\title{
Special issue on collaboration in software testing between industry and academia
}

\author{
Michael Felderer $^{1} \cdot$ Rudolf Ramler ${ }^{2}$
}

Published online: 9 November 2017

(C) Springer Science+Business Media, LLC 2017

Testing is the most widely practiced method to detect defects and to improve quality in the software industry. Many projects rely on testing as their primary quality assurance measure (Orso and Rothermel 2014). The practical importance of testing is also reflected by the often enormous budgets dedicated to this form of quality assurance (Huang and Boehm 2006). The academic interest is emphasized by a long and outstanding research history on static and dynamic testing of software systems (Orso and Rothermel 2014). Software testing is covered by dedicated academic conferences and events, tracks in major software engineering venues, and a wide array of scientific publications. Yet, how does this research impact the software industry? Despite the numerous ideas and approaches developed in academia, few research results have turned into practical applications that create a lasting benefit for practitioners. There is still a significant gap between the state of academic research and the state and needs of industrial practice. Bridging this gap is of mutual interest as collaboration between industry and academia supports improvement and innovation in industry and ensures industrial relevance in academic research.

This special issue focuses on collaboration between industry and academia in the context of software testing. It follows a series of events on industry-academia collaboration, such as the Workshop on Testing: Academia-Industry Collaboration, Practice and Research Techniques (TAIC PART) (Ramler et al. 2016; Alshahwan et al. 2015). The special issue comprises six papers that describe lessons learned from collaboration initiatives, share insights into the challenges and opportunities involved in the joint work of researchers and practitioners, and provide suggestions and approaches for better connecting the two sides.

The first paper Industry-academia collaborations in software testing: experiences and success stories from Canada and Turkey by Vahid Garousi, Matt M. Eskandar, and Kadir

Michael Felderer

michael.felderer@uibk.ac.at

Rudolf Ramler

rudolf.ramler@scch.at

1 Institute of Computer Science, University of Innsbruck, Innsbruck, Austria

2 Software Competence Center Hagenberg, Hagenberg, Austria 
Herkiloğlu analyzed a set of ten collaborative industry-academia R\&D projects in the area of software testing conducted by the authors based in Canada and Turkey. They assessed the involved challenges, patterns, and anti-patterns as well as their correlation with project success measures to, finally, provide evidence-based recommendations for ensuring success in industry-academia collaborations.

The second paper Toward the characterization of software testing practices in South America: looking at Brazil and Uruguay by Arilo Claudio Dias-Neto, Santiago Matalonga, Martin Solari, Gabriela Robiolo, and Guilherme Horta Travassos presents the results from a survey on software testing practices conducted with 150 practitioners from Brazil and Uruguay. The authors replicate related software testing surveys and other work on software testing practices from different countries. They were able to confirm and strengthen previous results regarding the use and importance of software testing practices.

In the third paper Model-based testing of digital TVs: an industry-as-laboratory approach, the authors Hasan Sözer and Ceren Şahin Gebizli describe their experiences in enhancing model-based testing for its adoption in the consumer electronics domain. They successfully applied the industry-as-laboratory approach, which they found highly effective for industryacademia collaboration and technology transfer. The approach has been used to define and refine research problems and to evaluate research results.

The fourth paper Test case selection in industry: an analysis of issues related to static approaches by Vincent Blondeau, Anne Etien, Nicolas Anquetil, Sylvain Cresson, Pascal Croisy, and Stéphane Ducasse explores practical issues and solutions for static test case selection in the context of large industry projects. The goal was to reduce the feedback time by automated testing for the developers of a company in the payments and transactional services industry. The study illustrates the challenging and exciting problems involved in realworld software testing.

The authors of the fifth paper Onboarding inexperienced developers: struggles and perceptions regarding automated testing, Raphael Pham, Stephan Kiesling, Leif Singer, and Kurt Schneider conducted a qualitative study on the skill gap between university graduates and industry expectations with respect to software testing. They surveyed and interviewed practitioners about their experiences with new hires. Results show that many practitioners are dissatisfied with the testing skills of newcomers and their negative attitudes toward automatic testing. New hires often have a hard time putting their theoretical knowledge into practice in a real-world environment, which requires companies to teach newcomers how to test from scratch.

Finally, the sixth paper SERP-test: a taxonomy for supporting industry-academia communication by Emelie Engström, Kai Petersen, Nauman bin Ali, and Elizabeth Bjarnason provides a taxonomy aimed to facilitate communication between researchers and practitioners collaborating in the area of software testing. SERP test is a Software Engineering Research and Practice taxonomy of testing that can be utilized for direct communication in industryacademia collaborations or indirect communication by making software engineering research more readily accessible to practitioners.

We would like to thank all the contributors for their hard work in preparing and updating the manuscripts. We would also like to thank the reviewers for their time and the effort in providing constructive feedback and improvement suggestions. Finally, we thank the Editorin-Chief, Professor Rachel Harrison, and the editorial staff for their support in getting this special issue ready for publication. 


\section{References}

Alshahwan, N., Felderer, M., Ramler, R. (2015) Industry-academia collaboration in software testing: an overview of TAIC PART 2015. ICST Workshops 2015, (pp. 1-2).

Huang, L., \& Boehm, B. (2006). How much software quality investment is enough: a value-based approach. IEEE Software, 3(5), 88-95.

Orso, A., Rothermel, G. (2014) Software testing: a research travelogue (2000-2014). Proceedings of the on future of software engineering (FOSE 2014) (pp. 117-132). New York: ACM.

Ramler, R., Felderer, M., Kitamura, T., Marinov, D. (2016) Industry-academia collaboration in software testing: an overview of TAIC PART 2016. ICST Workshops 2016, (pp. 238-239).

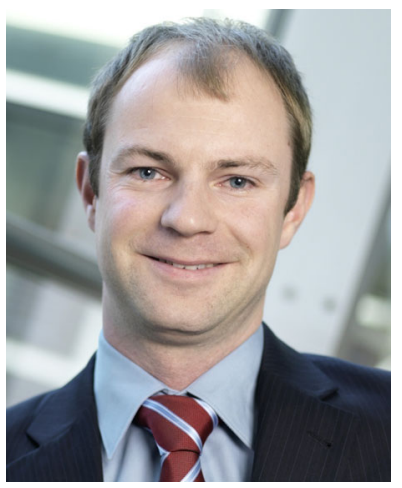

Michael Felderer is a senior researcher and project manager within the Quality Engineering research group at the Institute of Computer Science at the University of Innsbruck, Austria. He holds a PhD in computer science. His research interests are software testing and software quality in general, requirements engineering, empirical software engineering, software processes, and improving industry-academia collaboration. He works in close collaboration with industry and transfers his research results into practice as a consultant and speaker on industrial conferences.

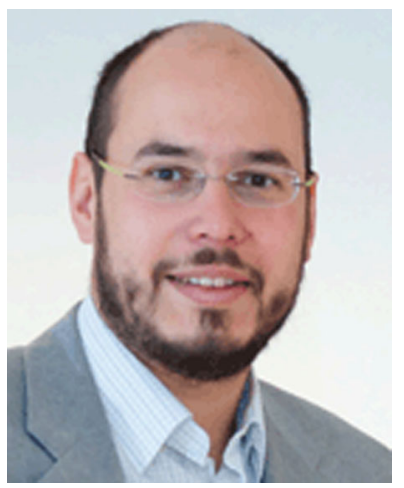

Rudolf Ramler is a researcher at the Software Competence Center Hagenberg, Austria. His research interests include software testing, quality management, and requirements engineering. He has led research projects as well as tool development for test automation, test management and quality monitoring. Rudolf works as a consultant in industry projects and is a lecturer with the Upper Austria University of Applied Sciences at Hagenberg and the 